\title{
Impact of the spectrum, location and interconnection between solar cells in the annual production of photovoltaic energies in photovoltaic concentration systems
}

\author{
J. Villa and A. Martí
}

\begin{abstract}
In this work we study the use of independently connected tandem solar cells (MJSC-IC) which are less sensitive to changes in the solar spectrum than series connected tandem solar cells (MJSC-SC). The efficiency of the maximum annual energy production has been calculated taking into account the variation in the spectrum and the efficiency of the cells. The results confirm the MJSC-IC have an annual energy production efficiency greater than the MJSC-SC. These suggest that it may be better to develop a 5-union MJSC-IC rather than increase the number of solar cells from 5 to 6 in the MJSC-SCs.
\end{abstract}

\section{INTRODUCTION}

Multi-gap photovoltaic systems have the capacity to exploit the solar spectrum better than photovoltaic systems with just one gap. Ideally, it is accepted that in multi-gap photovoltaic systems the solar cells that make them up absorb all of the photons with energies between their bandgap energy and the bandgap energy of the following solar cell. Likewise, it is assumed that every cell is transparent to those photons with less energy than the energy of the prohibited band of the said cell.

The concentration photovoltaic market is currently around $330 \mathrm{MWp}$ accumulated from installations connected to the electric network (grid), of which more than $90 \%$ are based on high concentration systems [1], [2]. The tandem concentration systems are made up of several solar cells which could be:

a) Series connected (MJSC-SC), having two terminal for all of the system.

b) Connected independently (MJSC-IC), accessing the terminal of each of them.

In order to maximise the efficiency of the photovoltaic conversion of the tandem solar cell system it has become necessary to optimise the energy values of the gap of the solar cells which make up the system. These values are matched to the spectrum achieving the best connection possible between the MJSC and the said solar spectrum, with which it is possible to extract the maximum power of the photovoltaic conversion system. The series connection in the MJSC-SC imposes a constraint in which the electric current that flows through the solar cells is the same. This restriction makes the operating point of each cell to become correlated with the others. In the MJSC-IC approach, the independent connection eliminates the constraint of the current, thus making the efficiency of the MJSC greater than that of those which are series connected, although at the cost of introducing the aforementioned terminals to each cell in the tandem.

In this context, the following questions are posed, and which this work attempts to answer:

a) The limiting efficiencies of the previously calculated tandem solar cells [3]-[6] must be reviewed as they were calculated making use of the now obsolete E891-87 [7] reference spectrum, or assuming that the sun provides radiation as a black body at $6,000 \mathrm{~K}$. In this work we use the new ASTM G173-03 [8] reference spectrum. The results of these revised calculations are detailed in section 2.

b) The MJSC-ICs have a lower sensitivity to the spectrum changes which also impacts on the annual production of energy. Furthermore, given that the MJSC-ICs are more efficient, it is worth considering the amount of additional annual energy that it is possible to produce with photovoltaic systems based on these cells in respect to those based on MJSCSCs. This study is detailed in section 3 .

\section{REVISON OF THE LIMITING EFFICIENCY}

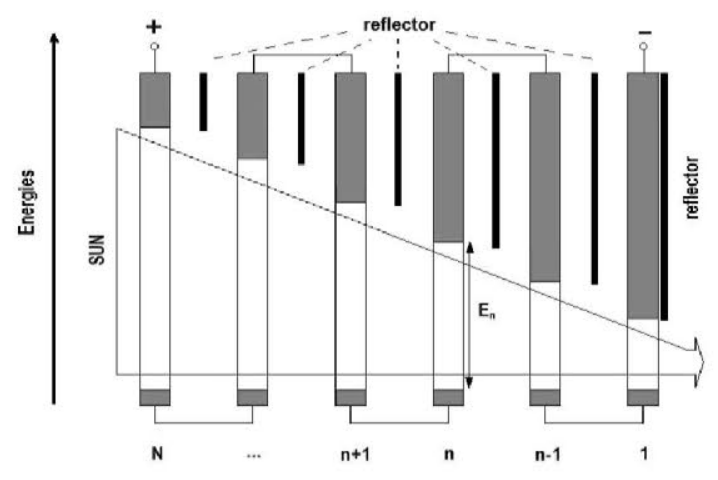

Fig. 1. Diagram of the series connected tandem solar cell system in this work. Mirrors have been placed between each solar cell and at the end of the system.

Fig. 1 shows the series connected tandem solar cell system (MJSC-SC). The independently connected system (MJSC-IC) is similar but two electric terminals are extracted from each 
cell. In order to calculate the limiting efficiency, each cell has a selective reflector attached to its rear part that avoids the radiative coupling between the cells. The theory related to the calculation of the efficiency limit of these systems was described in [3]. The calculations are based on the detailed balance theory formulated by Shockley and Queisser [9], [10].

The spectrum used in the calculations is known as ASTM G173-03 and has been calculated making use of SMARTS2 v.2.9.2 simulation software [11].
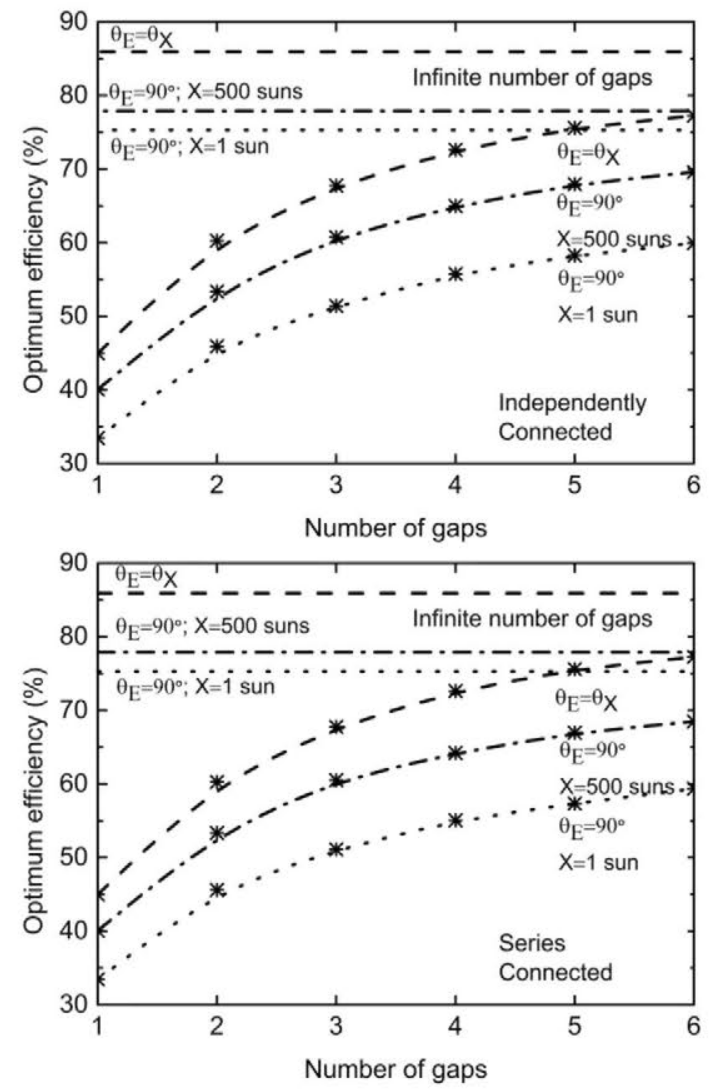

(b)

Fig. 1. Maximum efficiency as a function of the number of solar cells: (a) for independently connected cells in the tandem system; (b) for series connected cells in the tandem system.

Fig. 2 details the efficiency limits calculated for these spectra in this work up to a system consisting of 6 cells as well as for the ideal case of a tandem system of infinite cells for maximum concentration, 1 sun and 500 suns. The integrated power for the AM1.5 Direct Normal+Circumsolar irradiance conditions is $901 \mathrm{~W} / \mathrm{m}^{2}$ and for AM1.5 Global it is 1013 $\mathrm{W} / \mathrm{m}^{2}$.

As anticipated, the MJSC-ICs turn out to be more efficient than MJSC-SCs for the three illumination conditions studied. The relative difference in percentage between the conversion efficiencies of the MJSC-ICs and those of the MJSC-SCs for maximum concentration vary from $0.1 \%$ to $2.3 \%$. For a concentration of 500 suns, this relative difference in efficiency varies between $0.4-1.5 \%$. For a concentration of 1 sun, the relative differences go from $0.6 \%$ to $1.6 \%$.

The increase in the photovoltaic conversion efficiency is saturated when the number of solar cells that makes up the tandem systems increase taking, as previously demonstrated [12], [13], the same value for both types of MJSC of infinite solar cells, being, in accordance with the illumination conditions: $85.9 \%$ for maximum concentration, and $75.3 \%$ for a concentration of 1 sun and $77.9 \%$ for a concentration of 500 suns.

\section{EFFICIENCY OF ANNUAL ENERGY PRODUCTION}

The efficiency of annual energy production is defined as the annual PV energy produced divided by the total energy received from the sun. For this calculation it is necessary to know the efficiency of the solar cells as a function of the air mass of the solar spectrum (Fig. 3) [14], [15].

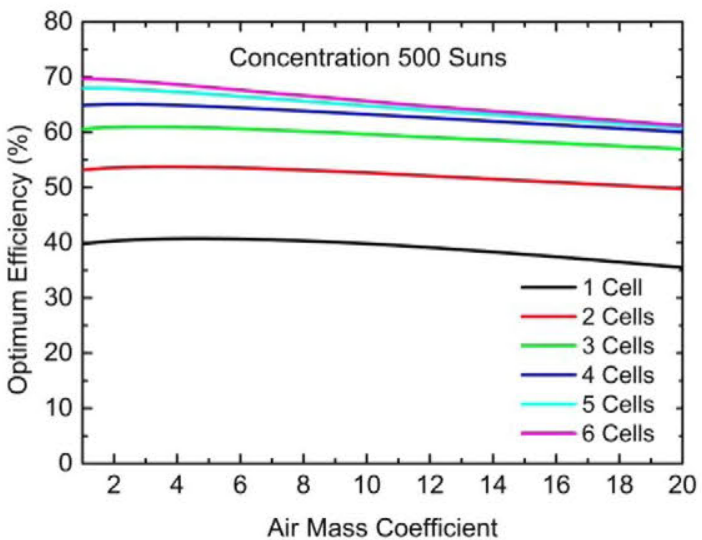

(a)

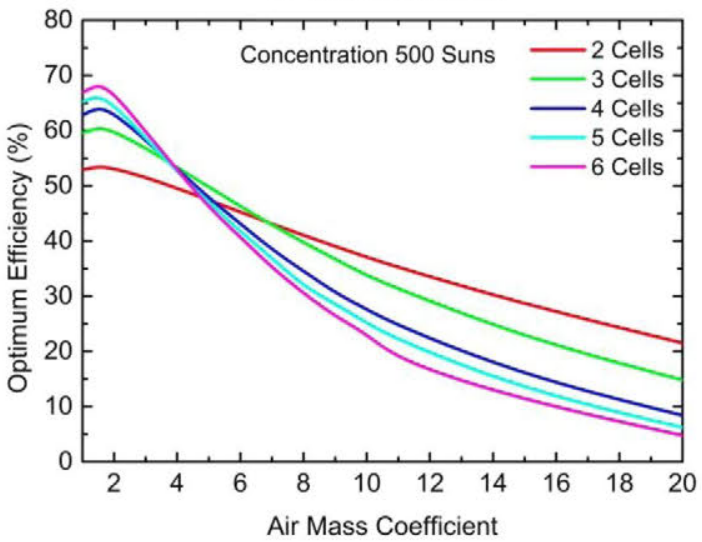

(b)

Fig. 2. Optimum efficiencies as a function of the variation in the solar spectrum for different air mass coefficients, $A M$, from AM1 to AM20, for the case of tandem systems with solar cells: (a) independently connected and (b) dependently connected for 500 sun concentration.

As example of the results, Fig. 4 details the values calculated for the efficiency of the annual energy for terrestrial latitudes of $\lambda=40^{\circ}$ and 500 suns. 


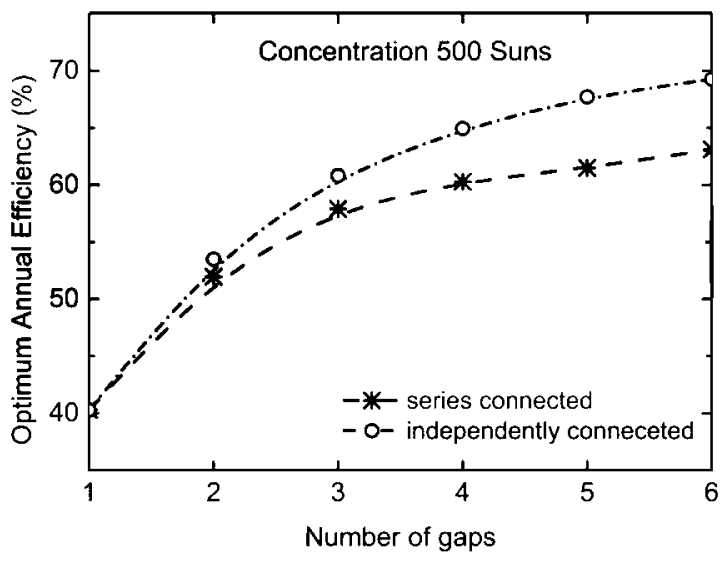

Fig. 3. Optimal annual efficiencies for stacked tandem systems with series connected and independently connected solar cells for terrestrial latitudes of $\lambda=40^{\circ}$ and a concentration of 500 suns.

Overall, the MJSC-ICs provide higher values of annual efficiency production than the MJSC-SC. Specifically, in the systems with 4 solar cells, under illumination conditions of 500 suns of concentration and for $\lambda=40^{\circ}$ terrestrial latitudes, it is more favourable to go from a system with series connection whose annual efficiency production is $60.3 \%$, to one with independent connections ( $64.9 \%$ ), which goes to an MJSC-SC of 5 solar cells $(61.5 \%)$. This same situation goes for the case of MJSCs of 5 solar cells, in which it is more favourable to go to an MJSC-IC of 5 solar cells $(67.7 \%)$ than an MJSC-SC of 6 solar cells (63.1\%).

In a more extended study we will include the results for the efficiency of the annual energy for 1 sun and maximum concentration.

\section{CONCLUSIONS}

The efficiency of annual energy production operating under the illumination conditions of 1 sun, 500 suns and maximum concentration for terrestrial latitudes of has been calculated. In this context, the MJSC-ICs have proven to have a greater efficiency of annual energy production as opposed to the MJSC-SCs, making an MJSC-IC of 4 gaps even more favourable than an MJSC-SC of 5 gaps.

The efficiency of photovoltaic conversion (calculating it for the new ASTM G173-3 reference spectra) for tandem systems that are made up of from 1 to 6 solar cells, both series and independently connected have been revised. Furthermore, calculations have been made for the illumination conditions corresponding to maximum concentration, 500 suns and 1 sun. With the aim of obtaining the photovoltaic conversion limit of a tandem solar cell system, the efficiency for a system made up of infinite solar cells has also been obtained.

The results demonstrate that the MJSC-ICs have a greater photovoltaic conversion efficiency than the MJSC-SC. On the other hand, the values of the gaps of the MJSC-ICs have a greater tolerance than for the MJSC-SCs.

\section{ACKNOWLEDGMENTS}

J. Villa is grateful for the financing from the BES-2013066463 aid, to the program financed by the Madrid Regional Government (Comunidad de Madrid) MADRID-PV (Grant S2013/MAE-2780), and to the Promesa (ENE2012-37804C02-01) project.

\section{REFERENCES}

[1] F. I. f. S. E. S. ISE, "PHOTOVOLTAICS REPORT 2014," Fraunhofer Institute for Solar Energy Systems ISE26 August 20152015

[2] S. Philipps, et al., "Current Status of Concentrator Photovoltaic (CPV) Technology," National Renewable Energy Laboratory (NREL), Golden, CO.2015.

[3] A. Martí and G. L. Araújo, "Limiting efficiencies for photovoltaic energy conversion in multigap systems," Solar Energy Materials and Solar Cells, vol. 43, pp. 203-222, 1996.

[4] M. A. Green, "Third generation photovoltaics: Ultra-high conversion efficiency at low cost," Progress in Photovoltaics: Research and Applications, vol. 9, pp. 123-135, 2001.

[5] S. Kurtz, et al., "A comparison of theoretical and experimental efficiencies of concentrator solar cells," in Proc 4th international conference on solar concentrators for the generation of electricity or hydrogen, El Escorial, Spain, 2007.

[6] S. Kurtz, et al., "A comparison of theoretical efficiencies of multi-junction concentrator solar cells," Progress in Photovoltaics: Research and Applications, vol. 16, pp. 537546, 2008.

[7] ASTM-International, "ASTM E891-87(1992), Tables for Terrestrial Direct Normal Solar Spectral Irradiance Tables for Air Mass 1.5 (Withdrawn 1999)," ASTM International, West Conshohocken, PA, 1992.

[8] ASTM-International, "ASTM G173-03, Standard Tables for Reference Solar Spectral Irradiances: Direct Normal and Hemispherical on $37^{\circ}$ Tilted Surface," ASTM-International, West Conshohocken, PA, 2008.

[9] W. Shockley and H. J. Queisser, "Detailed balance limit of efficiency of p-n junction solar cells," Journal of applied physics, vol. 32, pp. 510-519, 1961.

[10] A. De Vos, "Detailed balance limit of the efficiency of tandem solar cells," Joumal of Physics D: Applied Physics, vol. 13, p. $839,1980$.

[11] C. Gueymard, SMARTS2: a simple model of the atmospheric radiative transfer of sunshine: algorithms and performance assessment: Florida Solar Energy Center Cocoa, FL, 1995.

[12] A. S. Brown, et al., "Limiting efficiency for a multi-band solar cell containing three and four bands," Physica E: Lowdimensional Systems and Nanostructures, vol. 14, pp. 121-125, 2002.

[13] I. Tobías and A. Luque, "Ideal efficiency of monolithic, seriesconnected multijunction solar cells," Progress in Photovoltaics: Research and Applications, vol. 10, pp. 323-329, 2002.

[14] P. Faine, et al., "The influence of spectral solar irradiance variations on the performance of selected single-junction and multijunction solar cells," Solar Cells, vol. 31, pp. 259-278, 1991.

[15] J. Greif, The European solar radiation atlas vol. 1: Presses des MINES, 2000 